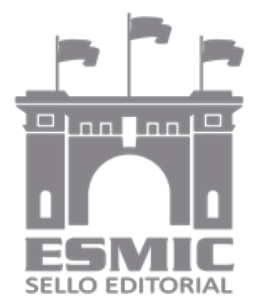

Revista Científica General José María Córdova

ISSN 1900-6586 (impreso), 2500-7645 (en línea)

Volumen 16, Número 21, enero-marzo 2018, pp. 35-59

http://dx.doi.org/10.21830/19006586.296

Citación: Ríos Cardona, M. P. (2018, enero-marzo). Elevator pitch, herramienta estratégica para proyectos de seguridad y paz en el posconflicto. Rev. Cient. Gen. José María Córdova, 16 (21), 35-59. DOI: http://dx.doi.org/10.21830/19006586.296. DOI: http://dx.doi. org/10.21830/19006586.296

\title{
Elevator pitch, herramienta estratégica para proyectos de seguridad y paz en el posconflicto
}

Sección: Derechos humanos y DeReCho INTERNACIONAL HUMANITARIO

Artículo de investigación científica y tecnológica

María Patricia Ríos Cardona*

Procuraduría General de la Nación, Bogotá, Colombia

Elevator pitch, strategic tool for security and peace projects in the post-conflict

Elevator pitch, ferramenta estratégica para projetos de segurança e paz no pós-conflito

Elevator pitch, un outil stratégique pour des projets de sécurité et de paix dans le post-conflit

Recibido: 16 de julio de 2017 • Aceptado: 28 de octubre de 2017

* https://orcid.org/0000-0003-3993-9544 - Contacto: mrios@procuraduria.gov.co 
Resumen. En el contexto del posconflicto que afronta Colombia, la competitividad de las instituciones es un imperativo para fomentar el desarrollo humano de una sociedad que se alista a crear un ambiente de paz, y que interpreta en esta última una oportunidad de desarrollo. Este artículo describe la técnica de comunicación asertiva, de síntesis y de persuasión conocida como elevator pitch. Asimismo, examina su utilidad para que los militares optimicen sus competencias en las tareas de apoyo y cooperación que deben emprender en el periodo de transición a la convivencia pacífica.

Palabras clave: comunicación asertiva; desarrollo y seguridad humana; liderazgo militar.

\begin{abstract}
In the context of the post-conflict confronted by Colombia, institution competitiveness is imperative to promote human development of a society prepared to create an environment of peace, that construes in the latter, an opportunity for development. This article describes the technique of assertive communication, synthesis, and persuasion known as, elevator pitch. It also examines its usefulness to optimize the military's competences in support and cooperation missions that must be undertaken in the transitional period towards peaceful coexistence.
\end{abstract}

Keywords: assertive communication; development and human security; military leadership.

Resumo. No contexto do pós-conflito que enfrenta Colômbia, a competência das instituiçóes é imperativo para fomentar o desenvolvimento humano de uma sociedade que se prepara para criar um ambiente de paz e que interpreta neste último uma oportunidade de desenvolvimento. Este artigo descreve a técnica de comunicação assertiva, de síntese e de persuasáo conhecida como elevator pitch. De igual modo, examina a sua utilidade para que os militares optimizem as suas competências nas tarefas de apoio e cooperação que devem desenvolver no período de transiçáo para a convivência pacifica.

Palavras-chave: comunicação assertiva; desenvolvimento e segurança humana; liderança militar.

Résumé. Dans le contexte de post-conflit, auquel la Colombie fait face, la compétitivité des institutions est un impératif pour promouvoir le développement humain d'une société qui se prépare à créer une ambiance de paix et qui voit en cette dernière une chance de développement. Cet article décrit la technique de communication assertive, de synthèse et de persuasion connue comme elevator pitch. Il évalue également son utilité afin que les militaires optimisent leurs compétences dans les tâches d'appui et de coopération qu'ils doivent entreprendre dans la période de transition vers la paix.

Mots-clés: communication assertive ; developpement et securite humaine ; leadership militaire. 


\section{El objetivo de un discurso no es necesariamente mover a los demás para que adopten tu idea, sino ofrecer algo tan atractivo que inicie una conversación.} Daniel Pink, estratega de la presentación.

\section{Estrategia de proyectos de desarrollo y paz}

El posconflicto exige activar una plataforma de componentes políticos, jurídicos, sociales, económicos y de desarrollo, en los cuales la pedagogía debe ser un elemento transversal. Asimismo, después de que un conflicto interno ha finalizado, se necesitan todas las herramientas disponibles para que los actores civiles, estatales, organizacionales y demás sectores involucrados con el avance integral para la construcción de futuro desarrollen sus iniciativas de paz.

En ese orden de ideas, y como agentes de gran importancia en los procesos de posconflicto, se habla de las capacidades, competencias y habilidades de las instituciones militares para garantizar la seguridad y el desarrollo humanos. En este contexto, los miembros del cuerpo constitucional armado deben estar comprometidos con optimizar los instrumentos de planeación competitiva, con el objetivo de crear espacios de confianza y fomentar iniciativas de emprendimiento que forjen el crecimiento en sectores debilitados por la confrontación armada. Sin importar cuáles sean los escenarios posibles después de la negociación del fin de un conflicto, e independientemente de su desarrollo, todo el cuerpo de un ente militar tiene que contar con las herramientas suficientes para aplicar sus destrezas en tareas puntuales de competitividad y crecimiento.

Precisamente, una de las destrezas competitivas que resultan útiles en la transición de un ambiente tenso a uno equilibrado, en el cual los militares van a aportar con tareas de apoyo y cooperación, es sin lugar a dudas la comunicación asertiva, específicamente, las habilidades de síntesis, interlocución, emprendimiento, aproximación y persuasión, necesarias para generar y promover proyectos que beneficien a las comunidades.

En tal sentido, en este artículo se describe la técnica de comunicación conocida como elevator pitch, su alcance e impacto comunicativo, y se describen las ventajas que como herramienta estratégica ofrece a la institución militar, por cuanto optimizaría la capacidad de síntesis y persuasión verbal, gestual y escrita de los militares para que logren captar, en un mínimo de tiempo, la atención de sus interlocutores — por ejemplo, autoridades institucionales, del sector privado, de las organizaciones civiles, líderes comunales y grupos sociales en general- y despierten su interés en las ventajas competitivas de un talento, un producto, una idea, una iniciativa o un proyecto de desarrollo y paz. El objetivo de esta técnica es preparar a las personas para que aprovechen al máximo un 
encuentro incidental y logren concretar una cita posterior en la que puedan exponer, ampliar y profundizar sus iniciativas y, en consecuencia, cerrar una negociación. En efecto, saber qué decir, cómo decirlo y a quién transmitirlo en periodos decisivos de transición del conflicto al posconflicto puede optimizar las oportunidades que se presenten, en aras de acceder a una exposición más amplia que conlleve la puesta en marcha de una idea de emprendimiento.

En ese sentido, este documento aborda, entonces, la comunicación asertiva, el liderazgo militar, el desarrollo y la seguridad humanas, por cuanto estos aspectos permiten interpretar el elevator pitch como una herramienta competitiva en escenarios de transición y posconflicto. Así, el interrogante que responde este artículo es el siguiente: ¿cuál es la importancia de la herramienta de comunicación elevator pitch para realizar las tareas de seguridad y desarrollo que precisa el posconflicto? Para responderlo, se plantearon tres objetivos específicos: i) describir el concepto de elevator pitch en el ámbito de la comunicación asertiva, ii) interpretar la construcción de futuro desde la capacidad de interlocución del liderazgo militar y iii) examinar el alcance estratégico de las herramientas competitivas en proyectos de seguridad y paz.

\section{Metodología}

Esta investigación tiene un enfoque descriptivo y analítico en el tratamiento de las fuentes, ya que analizar las dos categorías seleccionadas como eje conceptual, la comunicación asertiva —elevator pitch - y los proyectos de seguridad y paz para el posconflicto, permite entender el contexto en el que se aplicará la técnica y su grado de utilidad en la construcción de futuro. Por esta razón, el cumplimiento del objetivo general se basa en la riqueza interpretativa, la contextualización de los escenarios de seguridad y el análisis de la efectividad de esta técnica de interlocución y emprendimiento. Así, se puede afirmar que este enfoque metodológico propicia la exposición de las interrelaciones que establecen los actores, las actividades y las visiones de desarrollo que tienen los diferentes sectores de la sociedad, a la vez que facilita conocer el valor que el elevator pitch adquiere en el posconflicto. En esa medida, el análisis de los conflictos y del grupo de actores asociados a las iniciativas de desarrollo genera los insumos necesarios para identificar temas y acciones de común interés, en los cuales el elevator pitch puede desempeñar un papel importante en los proyectos de seguridad y paz.

Además, el enfoque descriptivo y analítico facilita la comprensión de las oportunidades que tiene el liderazgo militar para proponer y gestionar proyectos de seguridad y paz para el desarrollo y la paz en el posconflicto. En este contexto, la institución puede planear procesos de aprendizaje, asumir compromisos colectivos y, a partir del manejo del elevator pitch, avizorar planes de acción que incluyan propuestas para la construcción de futuro. Asimismo, este enfoque metodológico permite entender la orientación práctica de las estrategias de sinopsis, con las cuales el líder militar lograría persuadir a un interlocutor clave que pueda favorecer su idea o proyecto. 
En cuanto a las unidades de análisis, se debe mencionar que la investigación hizo el examen cualitativo de cuatro vectores: comunicación, gestión, liderazgo militar y competitividad. Finalmente, el protocolo de investigación consistió en hacer un diagnóstico de la comunicación asertiva con el objetivo de describir el concepto de elevator pitch. Posteriormente, y mediante un marco lógico de interpretación, se estableció el impacto de esta técnica en los proyectos de seguridad y paz. Así, para concluir, se describieron los principales sectores que pueden beneficiarse de su utilidad en los escenarios de posconflicto.

\section{Resultados y discusión}

A continuación se sintetizan los principales hallazgos de la investigación, según el plan descrito anteriormente.

\section{Elevator pitch en el ámbito de la comunicación asertiva}

\section{La comunicación asertiva}

Como parte del gran salto del tercer mundo al escenario del desarrollo, se exige la formación de personal altamente capacitado para el futuro, tanto en el sector público como en el privado, que esté preparado con herramientas y habilidades competitivas, fruto de un modelo educativo capaz de medir el porcentaje de progreso o regresión de los tecnólogos y profesionales en las diversas ramas de la productividad. Por su puesto, las instituciones militares no son ajenas a esta tendencia, así que, específicamente, en los proyectos de seguridad y paz en periodos de posconflicto, uno de los desafíos del liderazgo militar consiste en romper el círculo de falencias en el manejo del lenguaje y la interlocución con los actores comprometidos en la construcción de futuro. Es decir, los líderes militares deben desarrollar su capacidad de comunicarse en forma asertiva.

Pero, ¿qué es la comunicación asertiva? De acuerdo con Anguiano (2004), esta "implica el dominio de destrezas de interlocución e incidencia en los sectores competitivos" mediante "el estímulo de competencias para el logro de la persuasión, el impacto y la efectividad". En la comunicación asertiva son factores clave la claridad, la concisión y la rapidez, mediante los cuales las personas emplean eficientemente los recursos de la lengua hablada y escrita.

En el ámbito militar y en periodos de conexión social, la comunicación asertiva se mide por la aplicación de las competencias en tiempos justos y sectores específicos (Aguilar-Morales \& Vargas, 2010, p. 39). Al respecto, De Quijano et al. (1989) sostienen que los altos mandos militares comprenden que, en un periodo de posconflicto, los militares son percibidos como estrategas con estándares definidos de calidad y responsabilidad social, quienes además cuentan con un amplio manejo de la comunicación asertiva: "La comunicación asertiva en el ámbito militar exige dominio del planeamiento en condensación de mensajes y nitidez; decodificación y análisis de información; aplicación 
de habilidades asertivas en escenarios no probados; capacidad de interacción sostenible" (De Quijano et al., 1989, p. 121). Es decir, el estamento militar comprende no solo que estas necesidades - que hoy conforman la cadena estratégica de valor de un periodo de transición - deben afrontarse con un entrenamiento puntual que cumpla las exigencias del pensamiento visionario, sino, además, que de su adecuada administración depende la capacitación que necesitan los militares para incidir en el futuro.

Por su parte, Vaamonde (2001), Pérez (2001) y, más recientemente, Scheinsohn (2009) sostienen que la comunicación asertiva está conformada por técnicas que les permiten a las personas desplegar una plataforma de competitividad, sobre la cual podrán avanzar en los trabajos de desarrollo colectivo que se han planteado.

Las competencias de un determinado grupo, organización o líder que asuma el cometido de desarrollar actividades de promoción social y humana, precisan de las técnicas que forman parte de la comunicación asertiva y en las cuales es necesario tener claro su grado de utilidad. Estas piezas pueden ser la aplicabilidad de las habilidades duras y blandas, el elevator pitch, la gerencia de contenidos, los modelos de conexión social y la comunicación del liderazgo aplicado. Todas estas son un conjunto de técnicas que hacen de la comunicación asertiva una herramienta eficaz y propositiva y en la cual se exige dimensionar momentos de aplicación, poblaciones por trabajar, espacios y tiempos en aplicación de adiestramientos de cooperación. (Scheinsohn, 2009, p. 123)

Otras posiciones, como la del Centro de Estudios para el Desarrollo y la Democracia (Cepade) (Fisher et al., 2000), consideran el elevator pitch solo como técnica y herramienta que forma parte de la comunicación asertiva, entendida esta última como un instrumento integral. Asimismo, para el Cepade, las habilidades duras y blandas, así como la gerencia de contenidos, son per se un engranaje conjunto para la competitividad.

En todo caso, hay consenso en que la comunicación asertiva se enmarca en el trabajo que hoy demanda "la negociación". De acuerdo con la Fundación Friedrich Ebert Stiftung (2000), la comunicación efectiva es imprescindible en la negociación, ya que cualquier proyecto de promoción social y construcción de futuro implica aproximarse a los potenciales colaboradores para implementar las propuestas de desarrollo y crecimiento. En el caso de los proyectos de seguridad y paz en el posconflicto, no cabe duda de que establecer puentes de comunicación les exigirá a los líderes militares desarrollar un excelente manejo de estas competencias. Por tal razón, se debe enfatizar la importancia que tiene la comunicación asertiva y sus aportes para desarrollar canales de aproximación, cooperación de tareas y creación de propuestas de paz para el desarrollo.

\section{Elevator pitch}

Esta técnica busca abreviar la solidez del gran discurso y el argumento mediante el desarrollo de cuatro factores diferenciadores: quién propone, qué propone, para qué y para quiénes. Su objetivo es que los tomadores de decisiones se interesen en los proyectos que 
les propone su interlocutor en el breve tiempo que ofrece una oportunidad imprevista, es decir, despertar el interés del inversor o potencial colaborador, convencerlo de que acepte una cita formal para ampliar la idea y, de esta forma, lograr una negociación exitosa.

Para Faust y Faust (2004),

es una técnica que busca abreviar la solidez del gran discurso y el argumento, bien sea verbal o escrito, a fin de atrapar la atención de un inversor o un gestor de decisiones, de modo que este resuelva conceder una cita para ampliar la información de un proyecto. (p. 61)

Es decir, la utilidad del elevator pitch es que optimiza el tiempo para hacer que un interlocutor se interese en una trayectoria, una habilidad o una idea de negocio durante una circunstancia no prevista. Se trata de aprovechar la oportunidad del momento con un inversor inesperado, a fin de engancharlo y, posteriormente, concretar una reunión para profundizar en el tema de interés (Faust \& Faust, 2004, p. 78).

\section{$\underline{\text { Naturaleza }}$}

Conocida como el "discurso de ascensor", esta técnica fue promovida principalmente por la escuela americana, de allí que se relacione sobre todo con el campo comercial y de la negociación ${ }^{1}$. El término elevator pitch fue propuesto por el empresario estadounidense Philip B. Crosby, quien lo empleó por primera vez en la década de los ochenta para referirse a la forma de diálogo que se sostiene en los eventos que promueven la financiación de iniciativas empresariales, concretamente, aquel con el que se busca persuadir a un inversor sobre el atractivo de una idea durante un breve trayecto de ascensor. Sin embargo, el término solo ingresó a la plataforma competitiva cuando la Harvard Business School lo incorporó como herramienta estratégica para emprendedores (Báez, 2000).

Su origen yace en el mundo de los negocios, puesto que se refiere a comunicar, en el tiempo que dura el recorrido de un ascensor, una idea para que el interlocutor sorpresivo o inesperado se interese en el proyecto y el expositor logre concertar una cita o entrevista para ampliar los pormenores y las ventajas competitivas de esta. Igual puede referirse a una idea, un proyecto, un producto o a una iniciativa de emprendimiento en cualquier área que desee explorar logros derivados de crecimiento, bien en el campo financiero y económico, bien en el campo de la promoción social. (Kim \& Mauborgne, 2007)

1 No obstante, cabe mencionar que el origen del discurso sintetizado también se les atribuye a las dinastías chinas, que solían intercambiar mensajes casi que telegráficos, pero que captaban el interés de quienes deseaban persuadir (Kim \& Mauborgne, 2007). Al respecto, Vaamonde (2001, p. 29) considera que esta técnica pertenece a la filosofía china, basada en "la concepción del pájaro que con un solo trino sensibiliza, despierta los sentidos y abre horizontes". 
Por su parte, Harvey (2005) agrega que el elevator pitch

excede el mundo de los negocios y suele ser una excelente dinámica también para discutir políticas públicas, proponer ideas, fijar metas, desarrollar poder de síntesis y estar preparados para convencer a personas clave: "¿Qué le contarías en poco tiempo a tu ministro si te lo encontraras en un ascensor?". Existen recomendaciones y casos de éxito que guían en este camino de cómo desarrollar un arma de presentación de mi proyecto que siempre esté a mano y refleje realmente lo que quiero, para hacerle frente a cualquier encuentro en cualquier momento con las personas clave. (p. 156)

En cuanto a la aplicación del elevator pitch en los países de habla hispana, se puede afirmar que en España esta técnica forma parte de la empresa estratégica y cuenta con una marcada y continua pedagogía en las unidades militares. Respecto a América Latina, cabe mencionar que el caso más representativo es el de la empresa Embraer, uno de los cuatro fabricantes de aviones más importantes del mundo. Su fundador, Ozires Silva, un ingeniero oficial de la Fuerza Aérea brasileña, tuvo la oportunidad en 1969 de transmitirle su idea, mediante un elevator pitch, al entonces presidente Artur da Costa e Silva, que por emergencia aterrizó en São José dos Campos (Estado de São Paulo).

Como director de ese aeropuerto militar, Ozires recibe al presidente y en el ascensor tiene sus minutos de oportunidad: le comenta acerca del proyecto que tiene entre manos y logra captar la atención del mandatario. Ozires cuenta en sus libros cómo llamó la atención del presidente: "¿Usted sabía que aquí estamos en condiciones de fabricar aviones y venderlos al mundo?”, dicen que fue su pregunta provocadora. Así logró acordar una cita y convencerlo personalmente acerca del enorme potencial comercial del proyecto. Como la propuesta de desarrollar un biplano turbohélice no convencía al capital privado, después de su elevator pitch sobrevino la apuesta del gobierno brasileño y se fundó así Embraer. (Campero, 2012)

Sin embargo, es en la cultura norteamericana donde el elevator pitch cobra fuerza, pues la convierte en una herramienta de comunicación efectiva para poner en marcha un proyecto o para desarrollar una iniciativa, a partir de la precisión y la claridad de lo que se quiere aportar. Sobre este aspecto, Báez (2000) asevera que la técnica del elevator pitch se popularizó en los Estados Unidos en la década del cincuenta, cuando el país estabilizó sus relaciones con Japón después del ataque a la base militar de Pearl Harbor. El nuevo panorama social y económico después de la Segunda Guerra Mundial generó un intercambio tecnológico cada vez más intenso, el cual exigió a los estadounidenses gran precisión en la forma como abordaban los negocios con la cultura nipona, que se caracteriza porque aborda directamente el tema a tratar.

Asimismo, otros autores, como Friedman (2006, p. 34), subrayan que esta técnica de persuasión se gestó entre los administradores de la logística militar de los Estados Unidos, ya que "era preciso que cada comandante tuviera claro qué decir a su superior sobre el valor de una idea para su unidad cuando pudiera acceder a él por razones más 
casuales que programadas". De ahí que, en las bases del cuerpo armado, tanto en Canadá como en los Estados Unidos, se hiciera célebre y casi que imperativo explicar una idea en solo unos segundos.

En el caso concreto de su aplicación en contextos de posconflicto, tema de este artículo, es importante mencionar el trabajo de Ramonet (2004), quien analizó la experiencia de Kosovo (figura 1) y sintetizó los beneficios de aplicar esta herramienta en sociedades que están superando confrontaciones:

- Planificación del discurso útil.

- Logro del efecto de recordación.

- Administración de la virtud comunicativa para captar inversores.

- Manejo del factor sorpresa.

- Desarrollo de la persuasión visual, verbal, gestual y escrita.

- Potenciación de la virtud y el valor agregado del tecnólogo, el profesional o el facilitador para vender experiencia, producto, idea, iniciativa o proyecto.

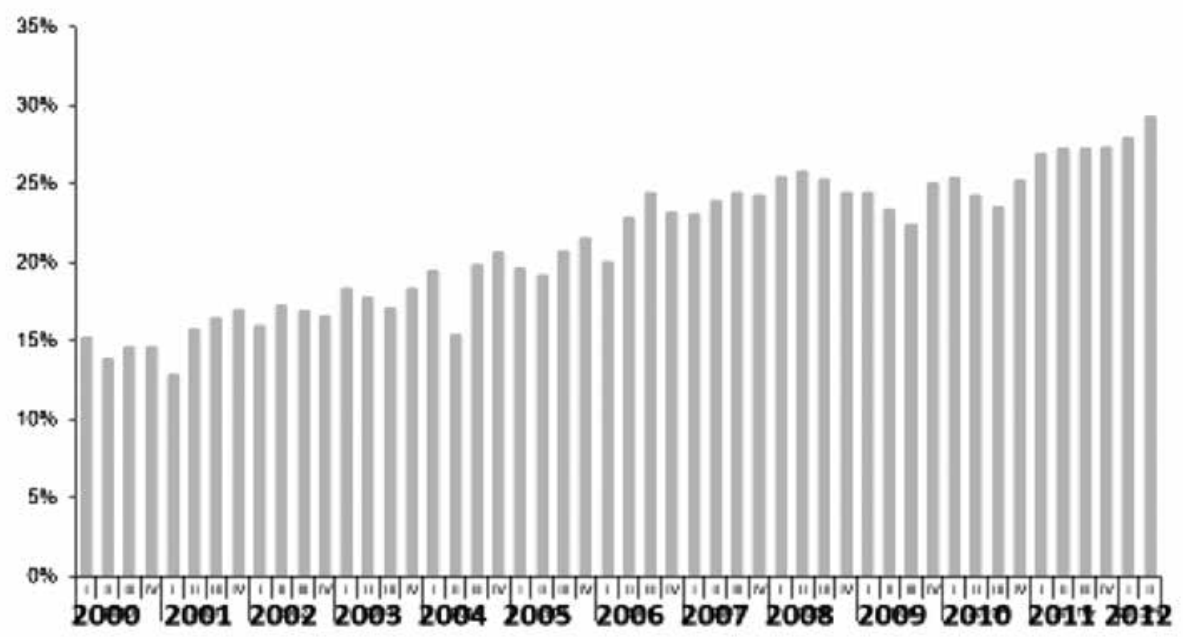

Figura 1. Elevator pitch por tasas de entrenamiento en unidades militares de paz en Kosovo. Fuente: Ramonet (2004).

En periodos de transición, los beneficios del elevator pitch cimientan las bases de la promoción humana, ya que favorecen la gestión de proyectos que satisfacen las expectativas y necesidades de seguridad humanas y proyección de futuro de las personas, así como sus necesidades cotidianas de supervivencia. Es decir, "cuando un gestor de desarrollo humano entiende la importancia de identificar en forma breve el valor agregado de una iniciativa de construcción de futuro, abre las puertas convenientes con las personas indicadas" (Celaya \& Herrera, 2007, p. 154). 


\section{Descripción del alcance e impacto del elevator pitch. El caso de Kosovo}

Como se mencionó anteriormente, la capacidad de síntesis y de persuasión, característica primordial del elevator pitch, la consagra como la técnica por excelencia de la comunicación asertiva. Con el objetivo de ofrecer unos derroteros de acción para que los miembros del ente militar la usen en un periodo de posconflicto, a continuación se describe el caso de Kosovo, un ejemplo claro de la reconstrucción de un país después de la guerra. Si bien en Colombia no se habla de un después de una guerra civil o étnica, como tampoco de una escisión de un territorio, la experiencia de esta nación balcánica sí le aporta al liderazgo militar información para que active sus competencias en proyectos de seguridad y paz.

La intervención militar de carácter humanitario realizada en Kosovo se define como "el uso de medios militares para alcanzar objetivos de carácter humanitario, como la protección de las personas no combatientes" (Hubert \& Bonser, 2002, p. 123). Como se sabe, la población albanokosovar fue objeto de una "limpieza étnica" por parte del gobierno serbio, por lo que la Organización del Tratado del Atlántico Norte (OTAN) tuvo que diseñar mecanismos para proteger los derechos fundamentales de la población civil, dado que la crisis de Kosovo representaba una amenaza a la estabilidad de Europa y a la seguridad de la población albanokosovar (Alonso, 1999).

Ahora bien, para Aguirre y Piris (2003), el reto que afrontó Kosovo, como en cualquier otro posconflicto, fue aprender a construir la paz y que el Estado, la sociedad civil, las Fuerzas Armadas y los grupos de contienda asumieran la responsabilidad de sostenerla. En este contexto, las tareas de asistencia humanitaria pusieron a prueba el conocimiento que tenían los miembros del Ejército sobre las iniciativas de cooperación civil-militar, en las cuales la evaluación que realiza cada actor a fin de realizar una tarea humanitaria digna, eficaz y eficiente fue definitiva para garantizar la seguridad humana (Delage, 1999). No obstante, el país balcánico logró transformar los puntos geográficos susceptibles de desarrollo integral, humano y sostenible, pese al difícil trayecto que desde su nacimiento han debido acometer como grupo humano (Delage, 1999).

En este aspecto, Thomashausen (2002) describe cómo el elevator pitch, en cuanto técnica y herramienta, condujo a que las autoridades locales se convirtieran en inversoras de proyectos liderados por militares en el área de la gerencia medioambiental y de provisión de recursos hídricos, para que los grupos humanos concentrados en áreas devastadas por la confrontación accedieran a los servicios básicos de saneamiento.

Como ejemplo del impacto potencial que tiene capacitar al cuerpo militar en elevator pitch, se presenta el caso de un miembro de los Cascos Azules de la Organización de las Naciones Unidas (ONU), quien expuso una breve presentación, de tan solo 20 segundos, con el fin de persuadir a las autoridades de Mitrovica (norte de Kosovo) para construir una red de acueducto en bien de los pobladores de ese territorio. Dicha exposición se efectuó en el marco de un evento pedagógico sobre trabajos de fuerzas conjuntas y cooperación mutua con los grupos sociales, ante una autoridad inesperada en el momento preciso. 
La prensa francesa, en entrevista con uno de los líderes cívicos, destacó la importancia de haber persuadido a las autoridades de poner en marcha la obra para bien de la comunidad y que, justamente, fuera un miembro del cuerpo de paz quien, simplemente exponiendo en un tiempo muy reducido el qué, el quién y los beneficios de esta, obtuviera para ese líder una cita con el gestor ambiental encargado de decidir el emprendimiento de las obras. Este quedó interesado con la ecuación formulada por el militar, que no rebasó más de 20 segundos ante la inesperada presencia del funcionario indicado para exponer la importancia del servicio: "Si el río Ibar abasteciera con buen acueducto 1.200 kilómetros a Mitrovica, los pobladores de la capital kosovar también podrían beneficiarse con 40 metros cúbicos más por habitante”. Entrevistado luego el líder cívico, explicaba cómo los cuerpos de paz de la ONU habían efectuado cálculos previos con ingenieros militares de la OTAN, por lo que el casco azul aprovechó la oportunidad inesperada para despertar el interés del gestor, quien no tardó en acoger la idea y conceder así la cita de ampliación de los detalles de la iniciativa. (Le Monde, mayo 2001; citado por Thomashausen, 2002)

También en Kosovo, la OTAN aplicó el elevator pitch en diversos proyectos en los que debió acordar tareas de apoyo para ampliar corredores humanitarios. Por esta razón, en los eventuales escenarios en los que se exigían informes de necesidades versus respuestas de parte de los líderes militares que trabajaban en los proyectos, fue necesario entrenar a cada jefe de comando de cooperación humanitaria para que supiera comunicar en poco tiempo, en forma verbal o escrita, la información precisa y relevante de su proyecto de apoyo comunitario, con el objetivo de que aprovechara los momentos en que coincidiera con un inversor social, una autoridad gestora o un cooperante óptimo.

En virtud de lo anterior, la funcionalidad del elevator pitch en las líneas de trabajo han sido hasta hoy las siguientes:

- Fortalecimiento y empoderamiento de las organizaciones para el desarrollo humano.

- Consolidación del proceso de incidencia política de estos logros.

- Continuidad del fortalecimiento del debate por la autonomía y el desarrollo local incluyente, así como avances en su concreción desde el nivel local.

- Conformación de alianzas que den vía a la construcción de proyectos conjuntos de desarrollo regional.

- Emprendimiento de acciones de generación de ingresos que conlleven la autosostenibilidad del proceso.

- Apoyo de procesos de paz en los ámbitos local, regional y nacional.

- Extensión del proceso a otros puntos geográficos del país y articulación con los intentos a punto de consolidarse.

Como casos concretos, en los territorios de Podujevo y Dakovica, Rieff (2004) relaciona una serie de situaciones en las cuales fue necesario "socializar en cuestión de minutos, y con el tiempo en contra, múltiples proyectos de seguridad humana ante la gente 
indicada en momentos no previstos para que los pobladores de puntos geográficos vulnerados por la guerra se beneficiaran con las decisiones de alta gestión" (p. 269).

Desde esa óptica, la prensa europea consignó como herramientas estratégicas todos los esfuerzos de interlocución que, desde las bases militares asentadas en los territorios de mayor complejidad tras la guerra, se registraban bajo el mando de los jefes de comando de proyectos de paz, así como de los líderes cívicos que debían articular tareas de coordinación entre las regiones, con la celeridad que las exigencias de reconstrucción demandaban. Los militares designados a las tareas de cooperación y avance para la convivencia pacífica pronto fomentaron el uso de la comunicación asertiva con sus subalternos. Rieff (2004, p. 270) lo consigna de la siguiente forma:

En Prizren y Urosévac, los jefes de las guarniciones de apoyo y cooperación se organizaban en grupos de diez para que, según su interacción con la comunidad, cada uno de ellos se ejercitara en comunicar lo mejor de la tarea que tenía asignada en los territorios y cuáles ventajas debían tener claras al momento de atender visitas de gestores de recursos e incluso de inversores de otros países cooperantes, las cuales, por el factor sorpresa, debían explicar en cuestión de segundos antes de que estos concluyeran sus visitas de inspección y así lograr interlocuciones posteriores para los coordinadores comunitarios o los emprendedores con los inversores y gestores a efectos de retroalimentar mejor a los interesados. Pronto cobró vigencia para el resto de los comandos de paz de los demás territorios kosovares la necesidad de que sus miembros se entrenaran en las técnicas de persuasión, entre las que sobresale el elevator pitch como una de las que más contribuyó a generar proyectos de cooperación y lograr que fueran ejecutables.

En este sentido, Colombia también tiene la capacidad de optimizar las competencias de sus líderes militares y las de los miembros del cuerpo armado para proponer y ejecutar proyectos de seguridad. Gracias a la ventaja competitiva de la comunicación asertiva, se podrían incluir las iniciativas dirigidas a los territorios que precisan de gestión en las políticas de cooperación, para que en el posconflicto se tomen en cuenta los puntos geográficos más vulnerables. Se sabe que incorporar la técnica como parte de las propuestas de nuevas estrategias no es tarea fácil, pero el caso de Kosovo muestra que es posible.

Asimismo, se debe tener en cuenta que implementar el elevator pitch en las tareas de cooperación y promoción humana en un periodo de transición tras el cese de un conflicto armado tiene un gran impacto en la gestión de la seguridad, dado que los proyectos de desarrollo humano y construcción de futuro necesitan inversores, administradores de la toma de decisiones y el visto bueno de las autoridades competentes. Por lo general, a estos actores de desarrollo les llega información a priori a través de un administrador de contenido, quien les transmite una idea condensada y puntual con el propósito de que pueda ser ampliada en una cita siguiente. Esto justifica que, en el caso concreto de Kosovo (figura 2), la comunicación asertiva sea considerada una pieza transversal en los entrenamientos militares que abordan las herramientas estratégicas para las operaciones de paz y la reconstrucción del país. 


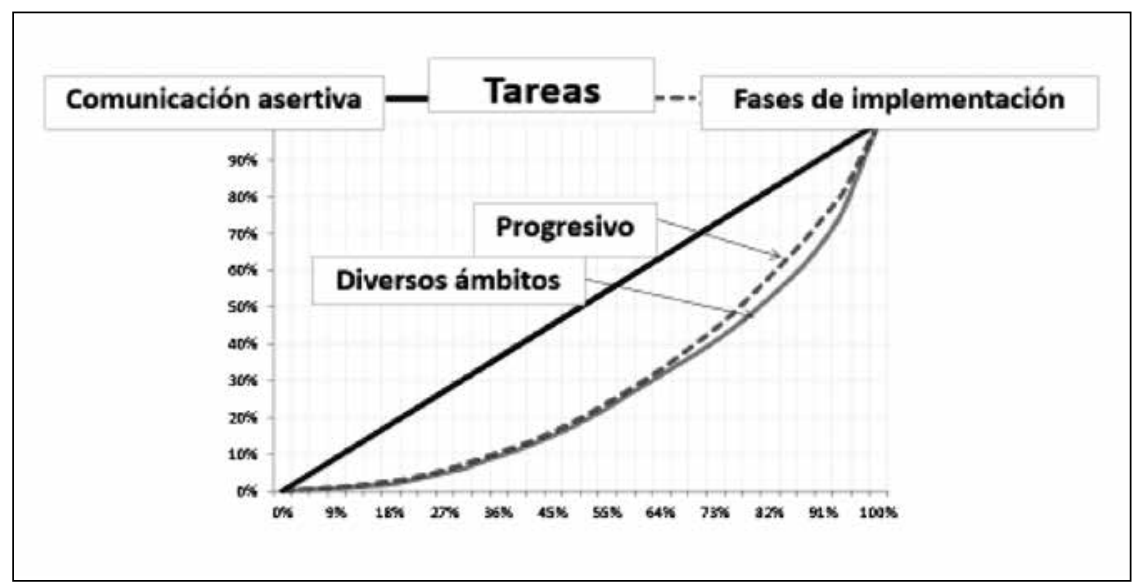

Figura 2. Porcentaje de desempeños de paz en Kosovo y la comunicación asertiva como eje transversal.

Fuente: elaboración propia con base en Thomashausen (2002).

Como se ve en la figura 2, los desempeños de paz durante la reconstrucción de Kosovo tienen altos porcentajes de avance progresivo en diversas áreas, ambos relacionados con la implementación de la comunicación asertiva como herramienta transversal.

\section{Análisis de la construcción de futuro a la luz de la interlocución del liderazgo millitar}

\section{Concepción preliminar}

En la construcción de futuro durante una fase de posconflicto, el desarrollo humano exige corresponsabilidad de los actores sociales y del Estado, puesto que la sociedad civil espera oportunidades de crecimiento, convivencia pacífica y seguridad. En ese orden de ideas, el líder militar debe ser consciente de que las destrezas de interlocución garantizan el éxito de sus tareas en el posconflicto, las cuales, derivadas de sus competencias, habilitarán proyectos de seguridad y paz.

Como el elevator pitch forma parte de esa comunicación asertiva que debe ser del dominio del líder militar en el posconflicto, es importante comprender el porqué de su aplicación y efectividad en la relación entre liderazgo militar y desarrollo humano. Con este propósito se debe escudriñar en la cultura de la paz, entendida como la necesidad de insertar los vectores de la convivencia pacífica en el imaginario y en la realidad de todos los actores del desarrollo de la sociedad (Stevenson, 2005).

Así, aplicar el elevator pitch exige que el líder militar tenga conocimientos en sistemas de gobernabilidad, apoyo logístico para negociaciones de paz, desarrollo de indicadores de desempeño, resolución de conflictos locales, gestión en capacitación de servicios del gobierno, conocimiento de logística de ayuda humanitaria, gestión estratégica para la pro- 
moción humana, entre otras habilidades y competencias del desarrollo colectivo (Juárez $\&$ Contreras, 2012).

Del mismo modo, la sostenibilidad en la construcción de futuros en el posconflicto exige del líder militar competencias que van más allá del apoyo a operaciones de paz. Estas competencias incluyen dominar las herramientas que soportan la ejecución de las tareas estratégicas con el fin de poner en marcha proyectos para la paz. Como asevera Stevenson (2005), gestionar el capital social demanda un liderazgo capaz de adaptarse a escenarios inesperados, criterio al que se ajusta el uso de la técnica del elevator pitch en el ámbito militar, en cuanto "hay percepciones estratégicas que el mismo nivel operacional y táctico desarrolla y que en determinado momento su aplicación y desempeño adquieren importancia en el entorno pacífico" (Harvey, 2005).

\section{La interlocución y la construcción de futuro}

El militar no solo deberá articular el liderazgo competitivo en el posconflicto, sino que también deberá tener en cuenta que el resultado de sus acciones únicamente será válido en cuanto transmita — a los grupos y colectivos con que trabaje- las herramientas de empoderamiento que, como el elevator pitch, operan en diversos ámbitos y disciplinas necesarios para construir la convivencia pacífica tras el cese de las hostilidades.

De ahí que la capacidad del militar para asumir conscientemente su papel en un escenario de posconflicto tenga el potencial de convertirse en una fortaleza, ya que su adiestramiento en los sistemas de gobernabilidad en poblaciones vulnerables, su apoyo logístico a negociaciones de paz en comunidades impactadas por la violencia, su manejo de indicadores de desempeño y su habilidad para detectar conflictos locales hacen que su pedagogía sea funcional y efectiva, no solo en logística de ayuda humanitaria, sino también en gestión estratégica para la promoción humana, entre otras habilidades y competencias del desarrollo colectivo (McRae \& Hubert, 2002).

Desde una visión más amplia, para la construcción de futuro también es necesario que el líder militar identifique los activos estratégicos del país, cifrados en los recursos naturales, la infraestructura, el capital social y sus valores - como la solidaridad y la cooperación mutua-, de manera que los transforme en un aporte sustancial en la reconstrucción de la paz. Asimismo, el líder militar debe ajustarse a la política de cooperación internacional, cuyos principios rectores se fundamentan en identificar agentes de cooperación, redireccionar los recursos en sectores de atención básica, madurar las fuentes de gestión, impulsar las competencias propositivas para el desarrollo y obtener recursos para sostener la gestión estratégica (ONU, 2009).

Con el desarrollo de estas habilidades, el líder militar no solo estará en condiciones de tomar las decisiones que le impone el contexto de posconflicto, sino que además logrará hacer compatibles las competencias militares genéricas — comandar, liderar, asesorar, administrar — con las necesidades reales del contexto de paz. 
Adicionalmente, para emplear el elevator pitch en el posconflicto con el objetivo de gestionar proyectos de seguridad y paz, el líder militar debe familiarizarse con la ecuación que hoy reclama la libertad humana en Colombia tras el cese del conflicto: "bienestar, desarrollo, calidad de vida igual libertad" ${ }^{2}$, que es el punto de referencia que el desarrollo humano tiene con los principios universales que consagran la libertad, el bienestar y la equidad como los derroteros esenciales de las políticas económicas y sociales de un Estado social de derecho. Dado que el liderazgo del militar gravita en las capacidades descritas, en las cuales comandar, asesorar y administrar son tareas de acción integral en el escenario de paz proyectado, Colombia está en condiciones de articular este liderazgo para activar el desarrollo humano nacional. Precisamente, es en esta misión en donde el elevator pitch se convierte en una herramienta de empoderamiento para gestionar cualquier proyecto de seguridad y paz.

Desde esa perspectiva, conviene, inicialmente, comprender que las variantes del desarrollo humano y su relación con las capacidades militares deben insertarse en un elevator pitch que busque eficacia en el momento de conectar a líderes comunitarios y grupos cívicos con inversores y gestores. En consecuencia, en el contexto actual del país el militar debe poseer capacidades de liderazgo que le permitan defender la soberanía, la integridad territorial y el orden constitucional con sus capacidades estratégicas bajo el lineamiento de la acción integral. Sin embargo, por el amplio espectro de amenazas que los escenarios de las hostilidades le han impuesto al cuerpo armado, su preparación para el posconflicto exige mayor optimización, perfeccionamiento y conciencia de sus competencias. Así, se espera que el líder militar las fomente en sus subalternos y las emplee como estrategia de empoderamiento para su adaptación a la convivencia pacífica, ya que desde hace sesenta años se ha dedicado a librar un conflicto armado interno (Monserrate, 2010).

Por lo tanto, en el momento de concebir proyectos de seguridad y paz, el miembro del cuerpo armado debe tener claro que la noción de desarrollo humano estratégico pertenece al concepto más general de desarrollo humano, que no solo atiende a la construcción de la seguridad como realidad en escenarios de confianza y equilibrio, sino que además es propio de sociedades que han retornado a la convivencia pacífica después de atravesar tensiones y confrontaciones armadas.

La seguridad humana como idea es un útil suplemento de la perspectiva expansionista del Desarrollo Humano, al prestar directamente atención a lo que a veces se denomina "riesgos negativos". Las inseguridades que amenazan la supervivencia humana o la seguridad de la vida cotidiana, o que ponen en peligro la dignidad natural del hombre y de la mujer, o que exponen al ser humano a la incertidumbre de la enfermedad y de la peste, o que someten a personas vulnerables a una penuria repentina causada por descenso de la actividad económica, exigen que se preste especial atención a los peligros de

2 En el Informe Mundial sobre Desarrollo, emitido por la ONU y la Organización para Estudios de Crecimiento Global, con sede en Zúrich, se planteó por primera vez una nueva dimensión de la libertad humana en la que quedaron definidos estos parámetros de percepción (Vancouver, 1997). 
la privación repentina. La seguridad humana requiere protección contra esos peligros, y la habilitación de la persona para que pueda enfrentarse con ellos y superarlos, de ser posible. (Rojas, 2006, p. 115)

De ahí que cualquier uso de una herramienta estratégica de la comunicación asertiva, bien se trate de la técnica elevator pitch o de la optimización de habilidades duras y blandas, precisa que el militar entienda que esta concepción del desarrollo humano, basado en la seguridad, implica su reconocimiento como derecho y condición necesaria para los Derechos Humanos, más aún en un contexto de confrontación armada. También debe comprender que Colombia está lesionada por una historia de inequidad y exclusión económica y social, la cual permea a tal punto el ejercicio político, que desestabiliza la capacidad de control del Estado en la defensa de buena parte del territorio. Al respecto, Van Creveld (2006) afirma que estas amenazas generalizadas o invasivas son la principal preocupación del Estado y que, en un proceso de transición al posconflicto, exigen la presencia del liderazgo militar, que tendrá que poner a prueba su capacidad de establecer interlocuciones efectivas.

Zaccaro (2002), por su parte, sostiene que los ejércitos de la mayoría de los países desarrollados desde hace tiempo están acometiendo diversos programas de modernización para mejorar las capacidades integrales del líder militar. En tiempos de paz, por ejemplo, "se requiere de la destreza que el líder militar acumula en inteligencia emocional y estrategia asertiva para incrementar la eficacia y eficiencia de las tareas de seguridad ligadas al desarrollo local, antes afectado por la supervivencia en medio del combate" (Van Creveld, 2006, p. 138).

En el caso de Colombia, las transformaciones que las Fuerzas Militares han tenido que realizar en este proceso de transición hacia la paz están forjando un liderazgo asociado inevitablemente al desarrollo integral humano. Por esta razón, las capacidades del líder militar, estrechamente ligadas a la disciplina, la responsabilidad, la lealtad, la reacción rápida, la anticipación de escenarios y la planeación de ámbitos de crecimiento, le conceden una ventaja competitiva que fundamenta su misión en el posconflicto.

Ahora bien, en este punto del análisis es necesario examinar la relación entre los conceptos desarrollo humano y liderazgo militar desde el legado del utilitarismo clásico. De acuerdo con Amartya Sen (2000), en una teoría de lo bueno y lo deseable es importante maximizar el bienestar colectivo, lo cual constituye una visión ética porque evita la discriminación injusta entre las necesidades humanas. Esta es la razón de que el Informe de Desarrollo Humano (IDH) plasme el nuevo enfoque de seguridad con base en las ideas de Sen (2000): el bienestar es la suma de las libertades humanas, y a eso es lo que se debe llamar crecimiento.

De acuerdo con la argumentación que se ha seguido hasta acá, el líder militar debe evolucionar su comprensión de la seguridad y del desarrollo humano como integralidad, lo cual se traduce en seguridad alimentaria, física, educativa, laboral, social, ciudadana, ambiental, cultural, política y militar. En tal sentido, estas áreas deben sustentar todo pro- 
yecto de seguridad y paz que el militar dimensione y que comunique aplicando la técnica elevator pitch.

Asimismo, cabe agregar que las exigencias actuales del crecimiento hacen que confluyan en un solo lugar las habilidades de negociación y el compromiso que tenga el líder militar para lograr el éxito en las tareas de desarrollo humano estratégico - específicamente en entornos de equilibrio y convivencia pacífica-, lo cual otorga validez a las acciones de emprendimiento y a las iniciativas de impacto (Fisher et al., 2000).

Continuando con las características del líder militar en contextos de posconflicto, también es relevante destacar las habilidades blandas, es decir, aquellas inherentes a la capacidad de interlocución y de optimización de las habilidades de comunicación innatas y no académicas. Este es, según Abarca (2004) y Eraso (2012), la esencia del liderazgo moderno: "La creatividad, la capacidad analítica y la agudeza anticipada del líder militar es la exigencia que los contextos competitivos están demandando" (p. 211).

Cuando el militar discrimina situaciones antes de tomar decisiones, sobre todo ante problemas en los cuales la persona pueda ser capaz de distinguir "lo esencial de lo accesorio", está aplicando habilidad blanda y sobre esta tienen que ceñirse las bases de su valor agregado en el liderazgo competitivo de hoy. No es solo el conocimiento y manejo de capacidades militares, sino las cualidades del líder militar en materia de interacción, actitud y búsqueda de salidas e iniciativas las que entran en juego para propiciar ambientes confiables, eficientes y con futuro, puesto que el crecimiento colectivo es reflejo de la maduración de un líder que, como el militar, es capaz de atenuar contextos de difícil confianza. (García-Guiu, 2012)

En este sentido, cuando Neef (1997) relaciona el crecimiento moderno con la capacidad de los individuos para entender el liderazgo como una plataforma de actitudes, se anticipa a lo que Van Creveld (2006) ha descrito respecto a las habilidades duras y blandas para ejercer el liderazgo, pero puestas a prueba con técnicas como el elevator pitch:

Las habilidades duras son herramientas técnicas que solo dan respuesta a escenarios ya probados y conocidos. Estas herramientas son: los conocimientos, inteligencia práctica, manejo de herramientas, poder de síntesis, de análisis, etc. Las habilidades blandas son todas aquellas herramientas que tenemos como seres humanos para la comunicación. Se trata de manejar un diálogo en el cual se puedan dirigir todos los puntos de vista de una manera constructiva para evitar conflictos. Tener la habilidad de dar distintas soluciones a un determinado problema, teniendo en claro la clave de este, para así actuar de la mejor manera. También es muy importante tener la empatía para así comprender los sentimientos y la forma de actuar de los demás. Tener la habilidad de descifrar cómo una emoción puede hacer actuar a alguien de una determinada manera. Y entre muchas otras, la habilidad de delegar; pero no solo eso, es conocer las habilidades de los otros para así poder confiar en el otro y en cómo desempeñará la tarea. Es muy importante tener la capacidad de fusionar las habilidades blandas y duras para procurar espacios, así como desarrollar las habilidades blandas buscando el equilibrio con las habilidades duras. (Van Creveld, 2006, p. 27) 
En contraste, para Lupano y Castro (2005), el liderazgo militar depende más del seguidor que del propio líder:

Desde las ciencias de la complejidad, definitivamente el liderazgo cambia radicalmente para erradicar mitos y creencias sobre lo que se piensa que es, para transformar al líder de un único ejecutor y tomador de decisiones a un transmisor de empoderamiento y distribuidor de conocimiento, creatividad y adaptabilidad, razones que hoy se les atribuyen a las habilidades blandas. (p. 168)

Finalmente, respecto a la construcción de futuro y a la luz de la interlocución del liderazgo militar, vale la pena reflexionar de qué manera las habilidades de los líderes militares tienen aplicación en los instrumentos que las Naciones Unidas, a través del concepto de operaciones de paz, utilizan para promover la paz y la seguridad internacionales antes, durante y después del conflicto armado:

1. Operaciones de mantenimiento de la paz o peacekeeping, las cuales están encaminadas a preservar la paz en países que tienen situaciones de tensión. Por ejemplo, en aquellos donde ya ha acabado la guerra con la firma de un acuerdo de paz y es necesario vigilar el cumplimiento de las condiciones establecidas. Estas operaciones requieren el consentimiento de las partes y se caracterizan porque los miembros del cuerpo militar solo utilizan la fuerza en defensa propia.

2. Operaciones de establecimiento de la paz, cuyo principio es pacificar una situación inestable y conseguir que las partes lleguen a un acuerdo global por medios pacíficos. Un ejemplo de estas operaciones fue la que llevó a cabo las Naciones Unidas en Namibia entre 1989 y 1990, o las que realizó posteriormente en El Salvador, Angola, Camboya y Mozambique.

3. Operaciones de imposición de la paz, que no cuentan con el consentimiento del Estado en el que se actúa, y contemplan el uso activo de la fuerza para imponer determinado mandato del Consejo de Seguridad. Como ejemplos se pueden mencionar los casos de Onusom II, en Somalia, y de Forpronu, en Bosnia y Herzegovina, Croacia, República Federativa de Yugoslavia (Serbia y Montenegro) y la ex-República Yugoslavia de Macedonia.

Estas experiencias y conceptos del liderazgo militar en tiempos de posconflicto son importantes porque ofrecen la posibilidad de interpretar en toda su dimensión las herramientas estratégicas de la comunicación asertiva, ya que sobre ellas reposa el éxito del empoderamiento de los proyectos de seguridad y paz.

\section{El alcance estratégico del liderazgo militar}

En la gestión humana, todas las tareas que competen a la dirección efectiva de personal deben abordar el desarrollo y la administración integral del talento humano desde 
todos los ángulos, de manera que propendan por la capacitación y el bienestar de quienes, desde sus áreas de trabajo, están llamados a dar respuesta a los desafíos que afrontan las instituciones y los grupos corporativos.

En efecto, cuando Greene (2007) establece un paralelo entre las mejores estrategias militares y cómo aplicarlas a la estrategia corporativa, remarca la necesidad de entender el sistema de gestión humana teniendo en cuenta la noción de competencia. Sin duda, este precepto, que rige hoy la competitividad moderna, cobra vigencia en el ámbito de la administración institucional en periodos de paz, de tal manera que el primer propósito que se debe definir en el contexto del posconflicto es redimensionar el proceso de administración del talento humano, ya que representa el resultado conjunto de la productividad (Ovalle, 2011).

\section{Equipos de alto rendimiento}

Como parte de la actividad estratégica y de apoyo a la organización en las entidades emblemáticas de los Estados, la "integración de la capacidad del talento humano conjunto" (Kim \& Mauborgne, 2005, p. 73) ha adquirido gran importancia. Parte de esta tendencia es el enfoque no lineal ${ }^{3}$ de la gestión moderna, que se centra en promover los equipos de alto desempeño porque "cada persona dentro del grupo tiene que tener claro su papel y debe dar lo mejor de sí para que el trabajo que tiene asignado sea elaborado de manera eficiente" (López, 2001, p. 121). Las Fuerzas Militares deben tener en cuenta este modelo para flexibilizar su gestión, de manera que incorporen las reglas de la competitividad moderna para empoderar proyectos de seguridad y paz mediante la aplicación de la comunicación asertiva —entre otras, el elevator pitch—. En ese sentido, el desarrollo de los conceptos asociados debe cifrarse en derroteros de eficiencia y prospectiva, puesto que en la medida en que se planee con rigor el acontecer actual, los miembros del cuerpo organizacional adquirirán mayor importancia para el posconflicto que la que tendría la modalidad de administración en sí misma. No obstante, es importante insistir en que cuando se trata del talento humano en la institución armada, el liderazgo se canaliza principalmente en la capacitación.

En Chile, por ejemplo, los miembros capacitados de las Fuerzas Militares trabajan en la reingeniería de las decisiones administrativas y son partícipes en las orientaciones que el mismo grupo, unidad o división resuelvan asumir. Esto significa que los grupos de alto rendimiento ejercen más un trabajo de supervisión que de gerencia clásica. $\mathrm{Al}$ respecto, Porter (1980) identifica los vectores que deben regir los grupos de alto rendimiento en la consecución de un objetivo:

3 Desde la estrategia de Napoleón, en la que la reorganización de las Fuerzas Militares francesas fue el punto de inflexión para el éxito en el combate, el enfoque no lineal se ha estado perfeccionando. En las fuerzas napoleónicas se precisaba actuar de modo fluido, rápido y no lineal, ya que la suficiencia flexible generaba la confusión del oponente. 
La búsqueda de buenos miembros o integrantes se efectúa en sentido directamente proporcional al talento individual para hacerlo coincidir con el talento grupal, bajo una estructura de trabajo y un radio de comunicación e interrelación del equipo, donde todos y cada uno reconozcan sus logros personales y grupales e identifiquen los recursos para poder trabajar en él, pues este es el punto de equilibrio y de flexibilización a donde el modus operandi de la buena gestión tiene que apuntar para la obtención de los mejores resultados, pero todo ello es posible si se toma en cuenta que la pluralidad de los procesos creativos redunda en la fusión de las capacidades operativas, donde, por ejemplo, los militares están dando muestra de combinación de talentos semejando un solo talento humano. (p. 56)

Es por esto que la integración del recurso humano debe propender, dentro del enfoque no lineal, a maximizar los talentos y los servicios, pero siempre basándolos en las capacidades habilitadas y certificadas. Aunque los equipos de alto rendimiento, entonces, se concentran en ahorrar recursos y esfuerzos significativos ${ }^{4}$, Ovalle (2011) subraya que el enfoque no lineal sugiere en sí mismo una paradoja: al maximizar el desarrollo de las capacidades del talento humano de una forma eficaz e integral entre instituciones con intereses comunes - o por lo menos similares_ y flexibilizar el espectro laboral, también pone a prueba las concepciones y la resistencia al cambio en pro del beneficio conjunto. Este es un aspecto que no deben soslayar las Fuerzas Militares en sus esfuerzos por modernizar la gestión castrense.

\section{El arte de gerenciar el talento humano}

Desde el marco conceptual expuesto, se entiende que, al flexibilizar la concepción del talento humano dentro de la institución militar, la toma de decisiones se relaciona estrechamente con los mecanismos, procedimientos, objetivos y resultados institucionales, es decir, con el direccionamiento de las Fuerzas Militares. Sin embargo, es necesario aclarar que la gerencia del talento humano parte de diseñar la reingeniería de recursos hacia una reorganización de desempeños, ejercicios y tareas conjuntas, de modo que el aspecto operacional adquiera la dimensión debida en el conjunto de las apreciaciones de mando y en los propósitos ejecutorios.

En cuanto a la estructura de la gerencia del talento humano, Kaplan y Norton (2003) enfatizan que esta debe distinguir los roles, para que estos, a su vez, tracen una sinergia específica. Así, aunar esfuerzos —en este caso los del Ejército, la Armada Nacional y la Fuerza Aérea-, cualquiera que sea el ejercicio de contención, es un reto que debe enfrentar cualquier grupo corporativo.

En este sentido, al aplicar el enfoque no lineal de cara a la competitividad global, la administración del talento humano en la institución militar debe adoptar una gerencia

4 Es el caso de las operaciones militares para la paz, en las cuales las fuerzas tienen definido su rol y lo hacen validar en el momento de ejecutar una tarea, de modo que la administración de esos talentos humanos se refleja en su óptima integración para el logro de un resultado eficiente. 
de las capacidades, soportada en un entrenamiento multipropósito que haga confluir la doctrina con las habilidades necesarias para cumplir los objetivos que la ruta productiva impone. En consecuencia, de las ventajas competitivas depende, en gran parte, que el enfoque no lineal del talento humano en la institución atienda las fortalezas individuales y grupales.

Desde esa óptica, la estructura de la organización es un medio que ayuda a la administración a alcanzar sus objetivos, los cuales son establecidos por la estrategia global de la empresa. En otras palabras, se podría afirmar que la estrategia debe estar alineada con la estructura de la organización. De ahí se parte hacia un enfoque contemporáneo que, desde la ingeniería del talento humano, se asocia con la toma de decisiones inteligentes (Hoffmann et al., 2002) y según el cual los oficiales líderes de unidades deben comprender que la eficiencia de la gestión de nuevos procesos reside, en gran parte, en la orientación asertiva de la administración de las herramientas. Todo esto con el objetivo de consolidar una ventaja competitiva, la cual se traduce en la optimización de costos para obtener una mayor ganancia en el cumplimiento de la misión y de los objetivos estratégicos.

De acuerdo con este modelo, las falencias de comunicación y las fallas en los procedimientos entre las divisiones, así como las disparidades e incomprensiones en el flujo informativo, afectan la sinergia del talento humano y el curso normal operativo y de apoyo, lo cual origina niveles de insatisfacción en algunos procesos de prioridad institucional. Esta situación hace necesario que en la gerencia del talento humano se agilicen las decisiones, se minimicen los tiempos de espera, se propicie una comunicación más fluida y se elabore una definición exacta de las funciones para que la institución alcance los resultados que espera de acuerdo con su estrategia.

Por esta misma razón, es fundamental que los líderes militares tengan claro los conceptos de estrategia, estructura, desarrollo y cultura organizacional, así como la interrelación entre estos, para que la institución dimensione los niveles administrativos del talento humano en las unidades, sobre todo de cara a las múltiples exigencias que deberán enfrentar durante el posconflicto.

Otro aspecto del concepto moderno de gerencia del talento humano es la acción del colectivo laboral, que debe estar dirigida a garantizar las actividades de diseńo y dirección de las capacidades, por cuanto los procesos deben ejecutarse de forma racional y coordinada con el objetivo de proveer elevada confiabilidad y agilidad, lo cual garantiza la preservación de la ventaja competitiva sostenible (Bowersox, 1979).

En la acción del colectivo laboral, los principios para definir criterios de eficiencia y desarrollar indicadores y modelos para evaluar la gestión humana son esenciales para el diagnóstico que guía la toma inteligente de decisiones, que, en el caso concreto de esta reflexión, significa definir la visión organizacional definitiva en la era de posconflicto.

En este sentido, el concepto de estrategia es determinante en el cambio organizacional y en la canalización del talento humano durante este periodo de transición. Definir con claridad la forma como se deben aplicar las comunicaciones asertivas, le permitiría a 
la institución militar no solo generar y fomentar proyectos de desarrollo y seguridad humanas, sino además aprovechar los factores externos o el entorno para transformarlos en vectores de cambio. Sin duda, al final, la visión que tenga la institución sobre la estrategia y la gestión del talento humano definirá la flexibilidad o rigidez de su diseńo organizacional (Chiavenato, 2009).

\section{Conclusiones}

La estructura del talento humano en la institución militar demanda que se sienten las bases de una gestión con sentido de oportunidad, beneficio y proyección. Asimismo, es necesario definir con claridad la estructura organizacional de las tareas que la institución asumirá en el posconflicto para beneficiar a la población.

Adicionalmente, los vacíos consecuentes de un periodo de confrontación deben convertirse en aciertos e integrarlos a la cultura corporativa de las operaciones de paz, en las cuales tendrán vigencia todas las herramientas estratégicas que construyan futuro y tiendan caminos de interlocución y articulación entre actores civiles, estatales, organizacionales y gestores o inversores de proyectos para el desarrollo de la seguridad humana. Según se ha afirmado en este artículo, el líder militar del posconflicto debe dominar las herramientas estratégicas y ponderar las ventajas competitivas de la institución en tiempos de paz, así como preparar y entrenar al personal subalterno para aplicar el elevator pitch, de tal forma que tenga la capacidad de desplegar su competencia asertiva para gestionar la seguridad y la paz, como se presentó en las experiencias de Kosovo.

Se concluye, entonces, que la ventaja competitiva del elevator pitch en los proyectos de seguridad y paz consiste en que empodera al líder militar para que fortalezca diversas iniciativas en los ámbitos de la seguridad humana: alimentaria, física, educativa, laboral, social, ciudadana, ambiental, cultural, política y militar. Con la aplicación del elevator pitch, como técnica que fomenta ese empoderamiento, la institución militar combate la duplicidad de procesos que pudiera presentarse en el posconflicto, ya que el talento humano del líder militar primaría en forma transversal a cualquier ejercicio. En este sentido, el talento humano, visto desde un enfoque no lineal, no solo debe contribuir a la resolución de problemas serios de estructura y procesos, sino que además tiene que alinearse con el objetivo estratégico impuesto por la institución en los proyectos de seguridad y paz.

En principio se pensaría que estos resultados no evidencian una alineación clara de la estrategia porque se tiene la idea de que las funciones y los roles de todos los actores del posconflicto están desorganizados y que esto ha influido negativamente en la gerencia del talento humano del militar del futuro. Sin embargo, en esta reflexión se aclara cómo empoderar a los líderes militares mediante el desarrollo de sus habilidades duras y blandas, con técnicas que, como el elevator pitch, fomentan la síntesis para que logren despertar el interés de inversores y gestores en iniciativas que favorecerán considerablemente a la población civil. 
En consecuencia, una de las propuestas iniciales de empoderamiento es incorporar el elevator pitch en la reingeniería del talento humano militar, de manera que se pueda ejercer un mayor control en los principales procesos que traería el advenimiento de funciones específicas en el planeamiento administrativo a corto, mediano y largo plazo de las operaciones de paz y los proyectos de desarrollo, en especial los procesos en los cuales las habilidades descritas son definitivas en la detección proactiva del recurso humano.

Por lo anterior, esta reingeniería de la gestión humana del líder militar permitirá, además de mejorar la eficiencia en los procesos, disminuir el impacto de los hallazgos encontrados por los entes de control. Así mismo, hará posible evaluar los riesgos y emprender las acciones correspondientes para que el talento humano militar avizore un futuro de eficiencia y competitividad.

\section{Agradecimientos}

La autora agradece a la Escuela Superior de Guerra y al Comandante del Ejército Nacional de Colombia.

\section{Declaración de divullgación}

La autora declara que no existe ningún potencial conflicto de interés relacionado con el artículo.

\section{Financiamiento}

La autora no declara fuente de financiamiento para la realización de este artículo.

\section{Sobre la autora}

María Patricia Ríos Cardona es abogada de la Universidad de Manizales, especializada en Derecho Constitucional. MSc en Seguridad y Defensa Nacionales de la Escuela Superior de Guerra. Labora desde hace 25 años en la Procuraduría General de la Nación y en la actualidad se desempeńa como Asesora de la Procuraduría Auxiliar para Asuntos Disciplinarios.

\section{Referencias}

Abarca, N. (2004). Inteligencia emocional en el liderazgo. Chile: Aguilar Chilena Ediciones.

Aguilar-Morales, J. E. \& Vargas-Mendoza, J. E. (2010). Comunicación asertiva. Network de psicología organizacional. México: Asociación Oaxaqueña de Psicología A.C.

Aguirre, M. \& Piris, A. (2003). Guerra por Kosovo. Anuario CIP - CIPIcaria.

Alonso, M. (1999). Serbia/Kosovo el segundo nudo y la baza desatendida. Viejo Topo, 128, 22-24. 
Anguiano, A. (2004). Comunicación asertiva [documento en línea]. Recuperado de http://www.intosol. com.mx/espacio/cont/gi/comase.htm.

Báez Evertsz, C. (2000). La comunicación efectiva. República Dominicana: Instituto Tecnológico de Santodomingo.

Bowersox, D. J. (1979). Towards total logistical management. In F. Wentworth \& M. Christopher (Eds.), Managing International Distribution. Gower Press.

Campero, E. (20 de octubre de 2012). La historia de Embraer y el "elevator pitch" [entrada de blog]. Recuperado de https://estebancampero.wordpress.com/2012/10/20/la-historia-de-embraer-y-elelevator-pitch/

Celaya, J. \& Herrera, P. (2007). Comunicación empresarial: la función de las nuevas tecnologías sociales en la estrategia de comunicación empresarial. Madrid: Grupo BPMO.

Chiavenato, I. (2009). Comportamiento organizacional. México D. F.: Mc Graw Hill.

De Quijano, S. D., Cornejo, J. M. \& Díaz, F. (1989). Asertividad y conflicto en la organización. Revista Psicología del Trabajo y de las Organizaciones, 13 (5), 68-80.

Delage, F. (1999). Del Tratado de Washington a la Guerra de Kosovo: la OTAN cumple 50 años. Cuadernos de Historia Contemporánea, 21, 341-353.

Eraso, D. (2012). El papel social del militar en el posconflicto colombiano. Bogotá, D. C.: Universidad Militar Nueva Granada, Facultad de Derecho.

Faust, B. \& Faust, M. (2004). Un CV más convincente: aprende a elaborar tu elevator pitch. Nueva York: Pearson Education.

Fisher, S. et al. (2000). Trabajando con el conflicto. Habilidades y estrategias para la acción. Guatemala: Centro de Estudios para el Desarrollo y la Democracia (Cepade) / Respoding to Conflict.

Friedman, T. (2006). La Tierra es plana. Barcelona: MR Ediciones.

Fundación Friedrich Ebert Stiftung. (2002). Manual del participante. Comunicación y negociación efectiva. México: Fundación Friedrich Evert Stiftung.

García-Guiu, C. (2012). El desafío de formar líderes militares. Recuperado de http://www.publicatuslibros.com/bibliotec/libro/el-desafio-de-formar-lideres-militares/

Greene, R. (2007). Las 33 estrategias de la guerra (3.a ed.). México: Editorial Océano.

Harvey, F. J. (2005). Liderazgo, actitudes y aptitudes. Revista de la Escuela de Comando y Estado Mayor del Ejército de EUA, 1.

Hoffmann, J., Sanchez, R. Micco, A., Pizzolitto, G. V., Sgut, M. \& Wilmsmeier, G. (2002). Port efficiency and international trade: port efficiency as a determinant of military cost. Maritime Economics \& Logistics, 5 (2), 199-218.

Horovitz, J. (1991). La calidad del servicio: a la conquista del cliente. Madrid: McGraw Hill.

Hubert, D. \& Bonser, M. (2002). Human security and the new diplomacy: protecting people, promoting peace. Montreal: McGill-Queen's University Press.

Juárez, F. \& Contreras, F. (2012). Liderazgo y complejidad. Conceptualizaciones e implicaciones para la organización actual. Espańa: Editorial Académica Española.

Kaplan, R. S. \& Norton, D. P. (2010). The Strategy-focused organization. Boston, MA: Harvard Business School Press.

Kim, C. y Mauborgne, R. (2007). La estrategia del océano azul. Bogotá, D.C.: Norma.

López Viñegla, A. (2002). Gestión estratégica y medición. El cuadro de mando como complemento del balanced scorecard. Madrid. 
Lupano Perugini, M. L. \& Castro Solano, A. (2005). Estudios sobre el liderazgo. Teorías y evaluación.

McRae, R. \& Hubert, D. (2002). Sécurité humaine et nouvelle diplomatie: protection des personnes, promotion de la paix (trads. Aude Burgo, Michael Buttiens y Betty Cohen). Quebec: McGill-Queen's University Press.

Mintzberg, H. (1989). Diseño de organizaciones eficientes. Buenos Aires: El Ateneo.

Monserrate, C. (2010). Metacompetencias: gestión de personas en tiempos de desconcierto. Madrid: Acción Empresarial.

Neef, M. M. (1997). Desarrollo a escala humana. Medellín: Proyecto 20 Editores.

Organización de las Naciones Unidas [ONU]. (2009). Componentes estratégicos para el desarrollo. Nueva York: ONU.

Ovalle Jaramillo, R. (2011). Integración del talento humano pensando en forma conjunta y coordinada. Revista Aeronáutica Fuerza Aérea, 264.

Pérez Aguilar, R. A. (2001). Estrategias de comunicación. Barcelona: Editorial Ariel.

Porter, M. E. (1980). Competitive strategy: techniques for analyzing industries and competitors. New York: Free Press.

Ramonet, I. (2004). La guerra de Kosovo y el nuevo orden mundial. En Ignacio Ramonet (Coord.), Guerras del siglo XXI. Barcelona: DeBolsillo.

Rieff, D. (2004). Kosovo: ¿el final de una época? En A la Sombra de las guerras justas: el orden internacional y la acción humanitaria (pp. 263-272). Barcelona: Icaria.

Scheinsohn, D. (2009). Comunicación estratégica. Buenos Aires: Granica.

Semler, S. W. (1997). Systematic agreement: a theory of organizational alignment. Human Resource Development Quarterly, 8 (1), 23-40.

Sen, Amartya. (2000). Desarrollo y libertad. Barcelona: Editorial Planeta.

Stevenson, J. (2005). Cero verdades a medias, el reto de rehacer la paz y sostenerla. Londres: Instituto de Estudios Estratégicos.

Thomashausen, S. (2002). The NATO intervention in Kosovo. En Sophie Thomashausen (Coord.), Humanitarian intervention in an evolving world order (African Century Publications Series n. ${ }^{\circ} 11$ ) (pp. 88-116). Sudáfrica: African Institute of South Africa.

Vaamonde, L. M. (2001). Técnicas de comunicación hoy (2.a ed.). Madrid: MacGraw-Hill.

Van Creveld, M. (2006). La transformación de la guerra. Buenos Aires: Editorial Uceda.

Zaccaro, S. J. (2002). Organizational leadership and social intelligence. In R. E. Riggio, S. E. Murphy \& F. J. Pirozzolo (Eds.), Multiple intelligences and leadership (pp. 29-54). Mahwah, NJ: Lawrence Erlbaum Associates. 